\title{
Improvement of the suture-occluded method in rat models of focal cerebral ischemia-reperfusion
}

\author{
PING ZHANG ${ }^{1}$, ZHEN HUANG ${ }^{1}$, HAI-QING YAN ${ }^{1}$, LIN-LIN SU ${ }^{1}$, \\ YONG-KUN GUI ${ }^{1}$, HAI-XIA LV ${ }^{2}$, BIN ZHU ${ }^{1}$ and TONG LI ${ }^{1}$ \\ ${ }^{1}$ Department of Neurology, the First Affiliated Hospital of Xinxiang Medical University, Xinxiang, Henan 453100; \\ ${ }^{2}$ Department of Pharmacy, Xinxiang Medical University, Xinxiang, Henan 453003, P.R. China
}

Received October 16, 2013; Accepted November 25, 2013

DOI: $10.3892 /$ etm.2014.1483

\begin{abstract}
The aim of the present study was to provide a simple method of establishing a rat model for focal cerebral ischemia-reperfusion (FCIR). The suture-occluded method was used to establish FCIR in male Sprague-Dawley rats. An incision was made over the bifurcation of the common carotid artery (CCA), through which a suture was inserted up to the internal carotid artery (ICA). The suture remained in the skin subsequent to model establishment and was withdrawn to the CCA to enable reperfusion. The reliability of the rat model was assessed via analysis of nerve function, tetrazolium (TTC) staining and pathological examination. Following FCIR in rats, the resulting neurological impairments were observed. TTC staining revealed infarcts and pathological examination revealed typical pathological changes. This modified method was simple, reliable and, therefore, may be used to investigate FCIR.
\end{abstract}

\section{Introduction}

Cerebral ischemic stroke has a high incidence of morbidity and mortality and results in a severe disability with a complex pathogenesis, which is unclear. Thus, this condition threatens the safety and quality of life of humans. Although the treatment for cerebral ischemic stroke has significantly improved recently, none of the available drugs for its treatment and prevention are considered to be optimal. The establishment of an optimal animal model, which simulates human cerebral ischemia may allow future investigations into the pathogenesis of, and prevention measures for, cerebral ischemia in humans.

Rats are selected as experimental animals due to their similarities to humans with regard to cerebrovascular anatomy and function. Furthermore, rats are of a standard species or strain, exhibit good intraspecific homozygosity and experimental

Correspondenceto: ProfessorPingZhang,Department of Neurology, The First Affiliated Hospital of Xinxiang Medical University, No. 88 Jiankang Road, Weihui, Xinxiang, Henan 453100, P.R. China E-mail: cnpingzhang@126.com

Key words: rat, focal cerebral ischemia-reperfusion, model repeatability and routine monitoring can be conveniently performed. There are numerous factors that influence the selection of rats as models; for example, oestrogen protects female rats from cerebral injury, therefore, male rats are included in investigations (1). Elderly rats are excluded due to the anatomical and pathological changes, which occur in their middle cerebral artery (MCA) and common carotid artery (CCA), such as tortuosity, luminal stenosis and arteriosclerosis (2). Furthermore, model establishment using adult rats leads to a relatively high success rate (3). Moreover, certain species of rats affect the model; for example, a small variation in the MCA of Fischer-344 rats results in a good consistency of cerebral infarct volume in the MCA occlusion (MCAO) model, whereas Wistar-Kyoto rats exhibit the smallest cerebral infarct volume and exhibit the largest variation. Characteristics of Sprague-Dawley (SD) rats fall between the abovementioned rat species; therefore, Fischer-344 rats are optimal for model establishment, however, they are rarely used as they are difficult to obtain. SD rats differ marginally from Wistar rats; however, intraoperative bleeding is minimal in SD rats, which makes surgery less complex. SD rats grow at a faster rate, are milder and less expensive; thus, they are often selected as experimental animals (4).

MCAO is the predominant cause of cerebral ischemic stroke (5) and the suture-occluded method is extensively used to establish MCAO models for performing clinical research (6). The conventional suture-occluded method established by Longa et al (7) is the prevailing method for the focal cerebral infarction model. However, it is complicated, time consuming and inevitably results in ischemic infarction of tissues (such as the temporalis, lingualis and pharyngeal muscles), to which the external carotid artery (ECA) supplies blood, which leads to further complications. To provide an optimal, reliable and simple animal model for FCIR injury research, a modified suture-occluded method of Longa et al was used. The experience of establishing a model using a modified process through anatomical observation of rats and an analysis of the key steps in establishing the model was also summarised.

\section{Materials and methods}

Animals. In total, 24 healthy, male SD rats (weight, $\sim 220-260 \mathrm{~g}$ ) were obtained from the Laboratory Animal Center of Southern 
Medical University (Guangdong, China) and were randomised into the sham-surgery $(n=12)$ and surgery $(n=12)$ groups. In each group, six rats were used for tetrazolium (TTC) staining and six to obtain tissue sections. The present study was conducted in strict accordance with the recommendations in the Guide for the Care and Use of Laboratory Animals of the National Institutes of Health. The animal use protocol was reviewed and approved by the Institutional Animal Care and Use Committee of the First Affiliated Hospital of Xinxiang Medical University (Henan, China).

Suture preparation. Nylon sutures (diameter, $\sim 0.2-0.25 \mathrm{~mm}$ ) were cut to a $60 \mathrm{~mm}$ length. One end of the suture was heated to form a smooth, spherical shape (diameter, $\sim 0.26-0.3 \mathrm{~mm}$ ), which was observed under a $\mathrm{BH} 2$ microscope (Olympus Corporation, Tokyo, Japan); the texture of the opposite end was rough. The suture was labelled with the required length, sterilised with alcohol and stored in heparin saline solution.

Establishment of the experimental model. The rats were fasted for $24 \mathrm{~h}$ prior to the experimental procedures, anaesthetised intraperitoneally using $10 \%$ chloral hydrate $(3 \mathrm{ml} / \mathrm{kg})$, fixed in a supine position and incised at the midline of the neck, (incision length, $20 \mathrm{~mm}$ ). The left CCA, internal carotid artery (ICA) and external CA (ECA) were exposed. The proximal CCA was ligated and the suture was suspended around the distal CCA for subsequent use, then the ECA was ligated at the bifurcation of the CCA. The proximal ICA was tied with a slipknot and the distal ICA was clamped using an artery clamp. A nylon suture was introduced into the ICA lumen through a puncture wound from a micro-incision, which was 5-mm distal to the CCA bifurcation. The slipknot was tightened and the artery clamp was removed. Insertion ceased once $\sim 19 \mathrm{~mm}$ of nylon suture had been inserted or until resistance was felt (where the tip of the suture reached the origin of the MCA), which resulted in the occlusion. The prepared suture was tied and fixed with a subcutaneously embedded thread residue; subsequently, the incision was closed. After $2 \mathrm{~h}$, the rats were anaesthetised with diethyl ether, all the occlusions were removed ( 2 needles) and the $10 \mathrm{~mm}$ nylon suture was withdrawn gently to reperfuse blood flow. In the sham-surgery group, $10 \mathrm{~mm}$ of nylon suture was inserted into the ICA lumen, and the remaining procedures were performed as described above. The intraoperative room temperature was maintained at $\sim 20-30^{\circ} \mathrm{C}$ and the postoperative anal temperature of the rats was $37 \pm 0.5^{\circ} \mathrm{C}$.

Neural function deficit score (NFDS). The neurological status of the rats was assessed $24 \mathrm{~h}$ following ischemia-reperfusion (I/R) according to the method described by Longa et al (7). The rats were classified into five grades: Grade 0 , no neurological impairment; grade 1, mild neurological impairment, failure to fully stretch the contralateral forelimb; grade 2 , moderate neurological impairment, rotation to the contralateral side; grade 3 , severe focal neurological impairment, toppling to the contralateral side; and grade 4, unable to walk spontaneously, exhibiting conscious disturbance.

Tetrazolium (TTC) staining. The rats were intraperitoneally overdosed with $10 \%$ chloral hydrate and were sacrificed by decapitation $72 \mathrm{~h}$ following I/R. Their brains were immediately harvested and frozen at $-20^{\circ} \mathrm{C}$. After $20 \mathrm{~min}$, five coronal sections $(2 \mu \mathrm{m})$ were incised from the upper forehead using a tissue slicer and incubated in TCC staining solution at $37^{\circ} \mathrm{C}$ for $30 \mathrm{~min}$. The sections were then fixed in fresh $10 \%$ formaldehyde for $\geq 24 \mathrm{~h}$.

Nissl staining. The rats were intraperitoneally overdosed with $10 \%$ chloral hydrate and sacrificed by decapitation $72 \mathrm{~h}$ after I/R. The chests were opened and the left ventricle (LV) was intubated; the blood was flushed with $4^{\circ} \mathrm{C}$ normal saline, followed by fixation of the $\mathrm{LV}$ with $4 \%$ paraformaldehyde phosphate buffer, overnight, at $4^{\circ} \mathrm{C}$. The $\mathrm{LV}$ was stored in 20-30\% sucrose phosphate buffer at $4^{\circ} \mathrm{C}$ until the tissue sank to the bottom of the solution. The temporal lobe was frozen and serially cut. The section was stained in Toluidine blue (Wuhan Boster Biotechnology Company, Wuhan, China) at $37^{\circ} \mathrm{C}$ for $5 \mathrm{~min}$, washed in distilled water for $3 \mathrm{~min}$. Conventional dehydration was then conducted using gradient alcohol and the lobe sections were mounted using a neutral balsam.

\section{Results}

Neurobehavioral observation. In the sham-surgery group, no neurobehavioral abnormality of the contralateral limb was observed: the rats were active and walked and drank well. Neurobehavioral impairments were evident in the surgery group: i) The rats exhibited hydroadipsia and paralysis of the contralateral limb, turned or rotated to the contralateral side, limped, and appeared dispirited and inactive (Fig. 1). ii) The results of the tail suspension test were positive, the rats failed to bend their contralateral forelimb when their tails were held, or were not able to independently move their contralateral limbs, and demonstrated spontaneous rotation to the contralateral side (Fig. 2). iii) The rats exhibited a decreased ipsilateral palpebral fissure and corestenoma (Fig. 3). A score of 1-3 indicated that the model had passed the test.

TTC staining. In the sham-surgery group, no infarcts were observed $72 \mathrm{~h}$ following I/R, whereas the surgery group demonstrated large areas of infarct, which were predominantly located in the parietal cortex and lateral striatum, or at the hippocampus and cerebral hemisphere. The left middle cerebral artery appeared white in colour, indicating that there was no haemorrhaging (Fig. 4). Furthermore, no apparent haemorrhaging was observed in the infarcts.

Nissl staining. The Nissl-stained histological sections were observed under a $\mathrm{BH} 2$ microscope. In the sham-surgery group, each layer of neurons in the cerebral cortex was densely arranged, specifically in layers II to VI. Occasional neuron distribution in layer I was observed. In layer V, the pyramidal cells were closely arranged and had larger cell bodies. A dense distribution of pyramidal cells was observed in the hippocampal CA1 region, which was divided into 2-5 layers. The cell contour was clear, with root-like apical dendrites that were apparent towards the radial layer (Fig. 5). In the surgery group, the ischemic injury involved the cerebral cortex, hippocampus, dentate gyrus and additional brain regions. The cerebral cortex presented six layers and the neurons were 


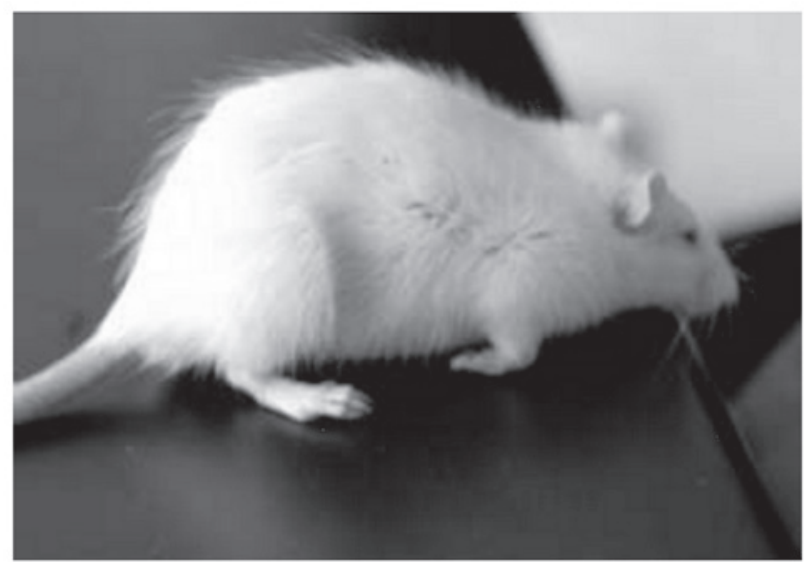

Figure 1. The rat exhibited an infarction in the left middle cerebral artery, with right limb paralysis and rotation to the contralateral side.

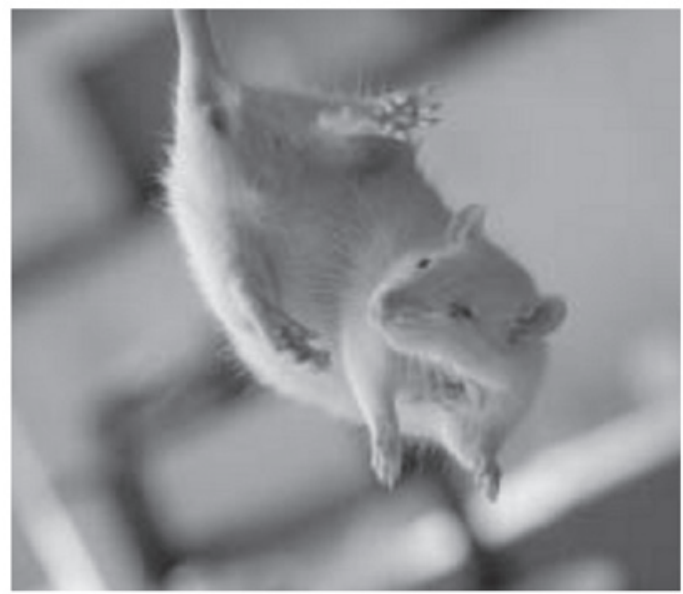

Figure 2. The rat exhibited an infarction in the left middle cerebral artery and the right upper limb and impairment of muscle strength was apparent.

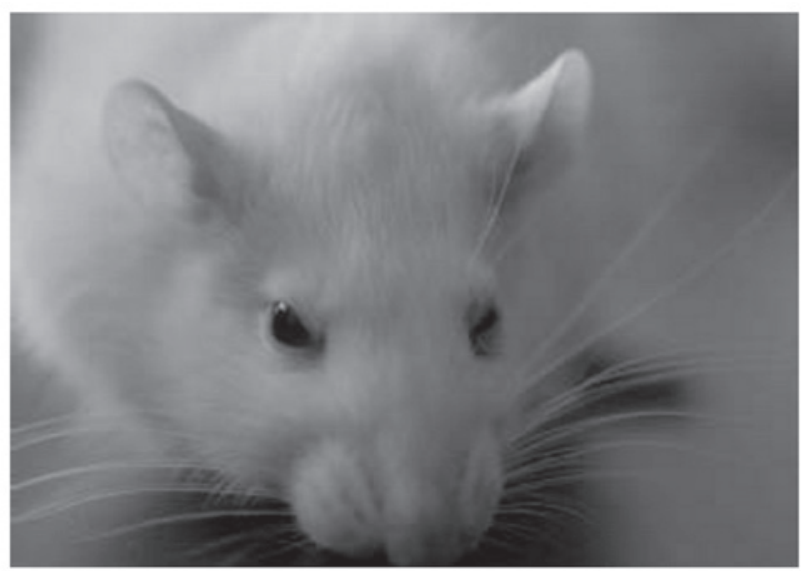

Figure 3. The rat exhibited a decreased palpebral fissure and corestenoma on the left side.

predominantly distributed in layers II to IV and exhibited a decreased density. The number of pyramidal cells in layer $\mathrm{V}$ was markedly decreased. In the hippocampal CA1 region, the pyramidal cell layer was thin, with a sparse distribution (Fig. 6).

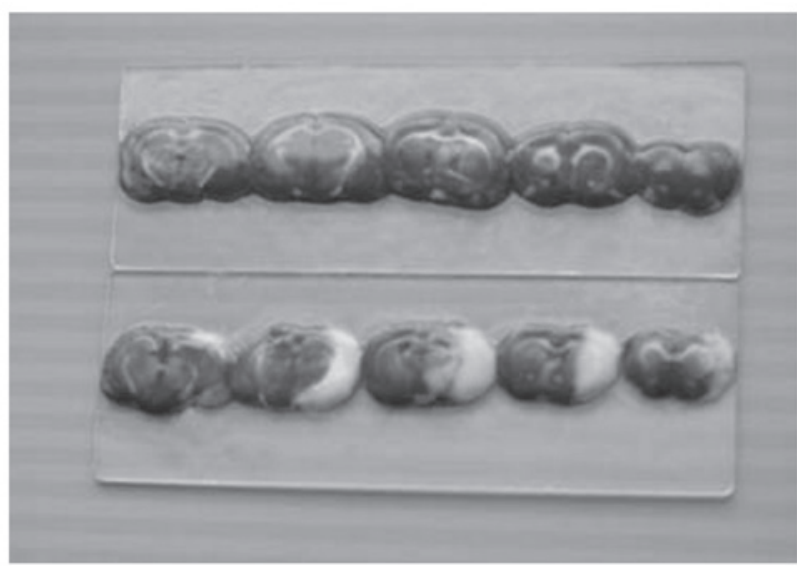

Figure 4 . The surgery group exhibited focal cerebral ischemia in the parietal cortex, with a morphology change in the hippocampal neuron. The left middle cerebral artery presented as white.

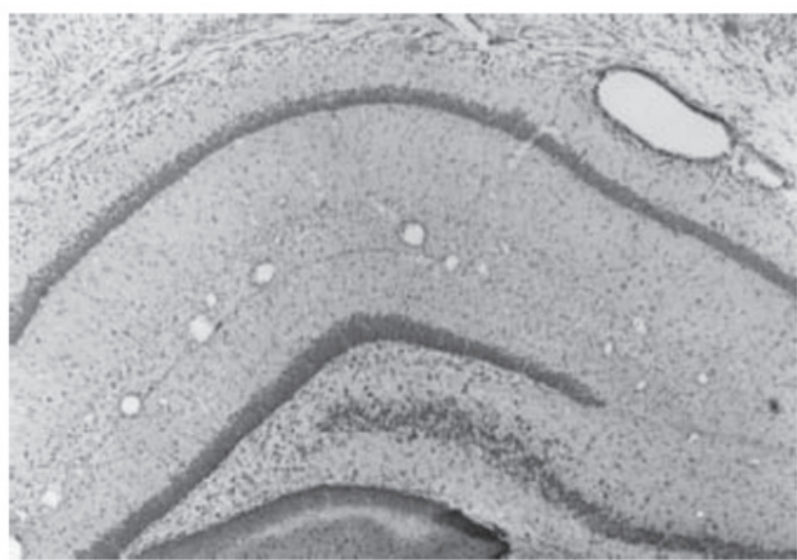

Figure 5. Nissl staining in the sham-surgery group. The neurons in the cerebral cortex and hippocampus were densely distributed (magnification, $\mathrm{x} 100$ ).

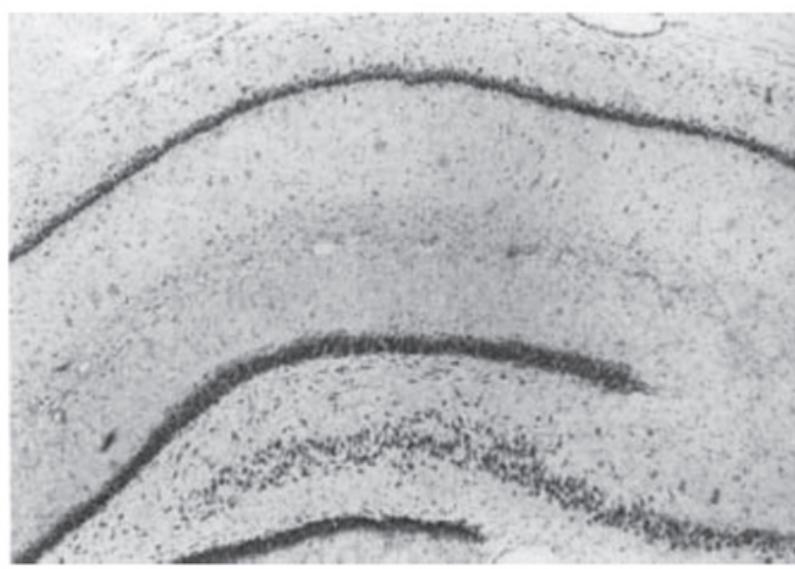

Figure 6. Nissl staining in the surgery group. The neuronal density of the cerebral cortex and hippocampus was decreased and the pyramidal cell layer was thinned (magnification, x100).

In the sham-surgery group, the neurons in the cerebral cortex demonstrated intact morphologies. The Nissl bodies appeared purple-blue in color, whereas the nucleus was light blue. The cells were plump, with evenly distributed Nissl bodies 

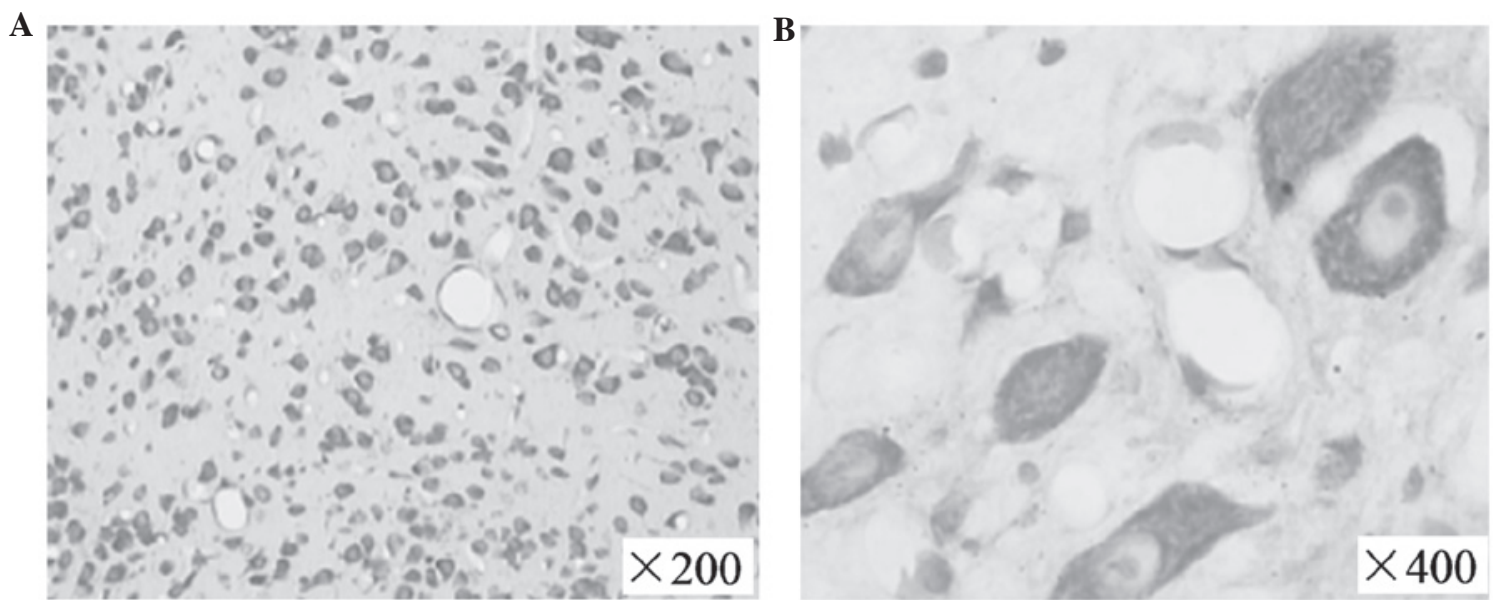

Figure 7. Nissl staining in the sham-surgery group, with the neurons in the ischemia cortex exhibited intact morphologies. (A) The spindle-shaped and (B) granular Nissl bodies were evenly distributed.
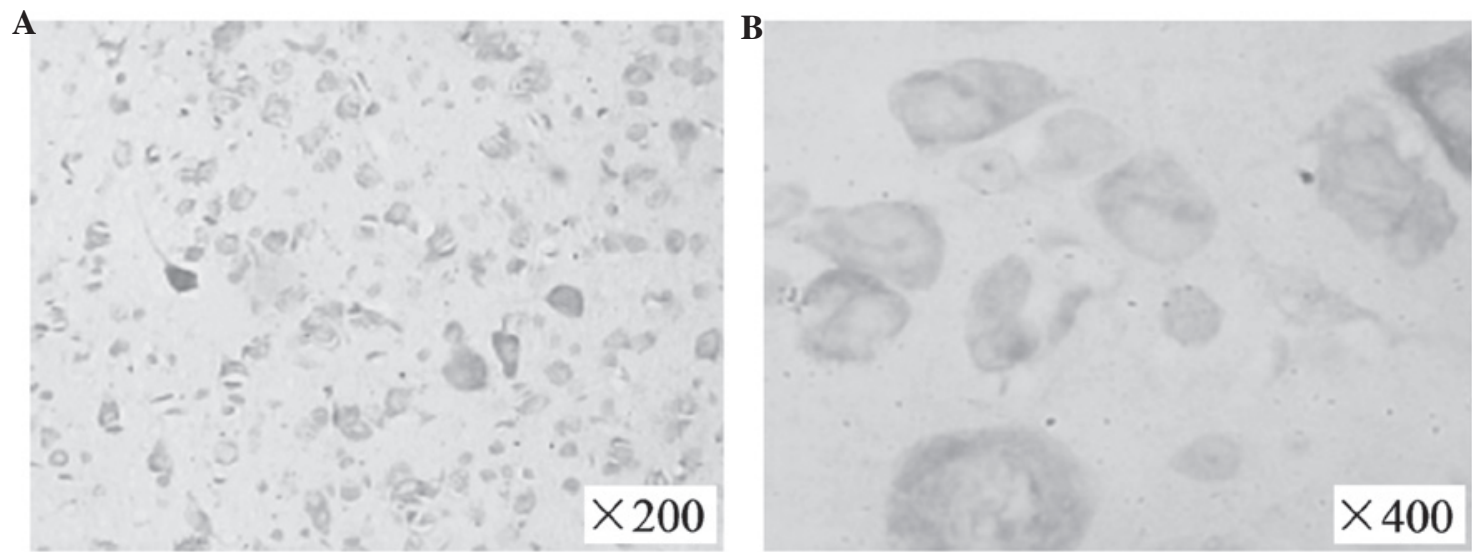

Figure 8. Nissl staining in the surgery group, $72 \mathrm{~h}$ following ischemia-reperfusion. (A) The Nissl bodies revealed a diffuse disappearance with residue in the individual cells. (B) The neurons were lost and cell ruptures were observed.

observed in the cytoplasm (Fig. 7). In the surgery group, the neurons in the cerebral cortex demonstrated ischemic changes, including decreased neuron density, shortened apical dendrites, swollen cells (which appeared to be ruptured), broadened intercellular spaces and neuron disintegration (Fig. 8).

\section{Discussion}

Ischemic cerebrovascular disease is a predominant human cerebrovascular disease and $>60 \%$ of cases frequently occur in the MCA. The cerebrovascular structure of rats is similar to that of humans in that the ICA and vertebrobasilar system form the Circle of Willis, which supplies blood to the brain through the arterial branches. Thus, rats are recognised as the optimal animal for establishing a cerebral ischemic model. The model is divided into global and focal cerebral I/R, according to the ischemic scope, or persistent and transient ischemia, according to the ischemia time. Among all of the animal models pertaining to strokes, the predominant model is MCAO, which is induced by focal cerebral ischemia in rats (8). MCAO can result in dysneuria, such as, typical limb hemiplegia and cerebral infarcts, which facilitate the observation and assessment of cerebral ischemia. Various methods of establishing a rat model of ischemia have been proposed including electrocoagulation, the suture-occluded method, cerebral embolisation, photochemically induced thrombosis and mechanical occlusion with craniectomy. The suture-occluded method is the commonly used approach to establish a cerebral infarction rat model (9-12), as demonstrated by Koizumi et al (10) in 1986 and improved by Longa et al in 1989 (7). The suture-occluded method without craniotomy is specifically suitable for investigations into FCIR injury due to the minor trauma area and the constant ischemic area (13). However, Longa's method requires separation and ligation of the ECA and the extracranial branch of the ICA-pterygopalatine artery as well as insertion of the suture into the ECA, which is complex to perform in rats due to the small surgical field, difficulty of separation, tendency for bleeding and the involvement of microinstruments; thus, its application is limited.

The suture-occluded method of Zea-Longa (7) was used in the present study to establish the FCIR model, with certain modifications: i) Incisions were made at the bifurcation of CCA to insert the suture from the CCA to the ICA, which avoided the insertion into the ECA and bypassed the bend 
at the CCA bifurcation; thus, suturing was straightforward. ii) Cerebral infarction severity was not affected by ligation of the pterygopalatine artery. The suture was mistakenly inserted into the pterygopalatine artery, however, the resistance was felt after inserting $10 \mathrm{~mm}$ of suture; thus, it was withdrawn and adjusted to a different angle, which was consistent with the study (8). iii) The suture was subcutaneously embedded following establishment of the models; reperfusion was conducted under rapid anaesthesia by unpicking one to two stitches, withdrawing the suture to the CCA, cutting the suture near to the skin and closing the incision. This generally avoided vessel injury, on removal of the suture, as well as movement and slippage of the suture that resulted from movement of the rat following revival, which enhanced the success rate and reliability of the model. iv) Additional surgery did not influence the healing; rapid anaesthesia with diethyl ether enabled the reperfusion process to be completed in 5 min without altering the experimental conditions, which ensured homogeneity of the model. However, there were numerous factors that affected the model: the surgical process was standardised as it was performed at the same time each day, the rats were randomised into different groups, the age, gender and body weight of the rats was consistent, in addition to maintaining a constant diameter of the nylon suture and the spherical shape at the ends.

In vivo evaluation of a successful rat model includes monitoring the MCA blood flow and electroencephalograph, distinction between cortical and subcortical infarction by positron emission tomography and magnetic resonance imaging (MRI), detection of the cerebral ischemic core and determination of the ischemic penumbra $(14,15)$. Instrument monitoring is accurate, however, it is expensive and limited by the experimental conditions; specifically when a large quantity of animals are investigated. An optimal NFDS system should be non-invasive, low cost, reliable, time saving and straightforward to perform. The symptoms of focal cerebral ischemia are characterised by contralateral limb dysfunction; therefore, the neuropathy symptom score is internationally regarded as a general criteria. Numerous scoring methods exist including, the Bederson method (16), the Nagaoka method (17), the Longa method (7), the Tatlisumak method (18) as well as the Hattori method (19). Previous studies have confirmed that the NFDS is able to evaluate MCA infarction due to the close association between them, moreover, behavioural scores have been demonstrated to correlate with the cerebral infarct volume (20). During the establishment of the MCAO model using numerous SD rats, Boyko et al (21) identified the association of the behavioural score with the infarct size and demonstrated the correlation between the predicted and actual values using MRI. Neurobehavioral analysis, independent of any devices, is a valuable, effective, reliable and sensitive method of evaluating the nervous system. Numerous scholars have suggested increasingly comprehensive evaluation methods, including the scoring of neurological and vestibular function in ischemic animals, which results in an improved assessment. The NFDS by Longa et al, was used in the present study and the model was labelled as successful if the rats accorded with any indicators of the scoring system. However, ipsilateral Horner's syndrome resulted from the intraoperative injury of the superior cervical sympathetic nerve, which resulted in a decreased palpebral fissure and corestenoma and, therefore, failed to serve as an individual sign of a successful model.

The surgical principle of the FCIR model was: The suture (length, $19 \mathrm{~mm}$ ) in the ICA simultaneously blocked the two sources of arterial blood in the Circle of Willis; the ipsilateral ICA and the posterior communicating artery that connects with the vertebral artery, while the ipsilateral anterior CA maintained the ability to obtain blood from the contralateral ICA. On withdrawal of the suture to the ICA, the ischemic area was reperfused through the contralateral ICA and vertebrobasilar artery of the Circle of Willis. The ischemia time was $\sim 2-6 \mathrm{~h}$ in the rat cerebral I/R injury model. The interaction between the four physiopathologic mechanisms, including toxicity of excitatory amino acids, peri-infarct depolarisation, inflammation and cell apoptosis following I/R (22), further aggravating the symptoms of ischemia and reaches a steady state within 3-6 h. Kawamura et al (23) demonstrated that ischemia time, which was $>3 \mathrm{~h}$, resulted in the reperfusion rats exhibiting comparable neurologic manifestations, cerebral infarct volume and encephaledema to those rats without reperfusion following $24 \mathrm{~h}$ of ischemia. Ischemia duration $<1 \mathrm{~h}$, led to reperfusion resulting in reduced infarcts and greater variation, an ischemia duration of $2 \mathrm{~h}$ falls between the abovementioned conditions. Therefore, reperfusion was performed following an ischemia duration of $2 \mathrm{~h}$. In addition, cerebral edema peaked three days after the clinical cerebral infarction; thus, TTC staining was conducted on the third day.

In conclusion, the results of the present study revealed that the modified rat MCAO model exhibited evident indicators of neurologic deficit and resulted in constant infarct locations. The sham-surgery group demonstrated integral brain structure without ischemic variation, and no infarcts were observed following TTC staining, which further demonstrated the reliability of the model.

\section{References}

1. Simpkins JW, Wen Y, Perez E, Yang S and Wang X: Role of nonfeminizing estrogens in brain protection from cerebral ischemia: an animal model of Alzheirmer's disease neuropathology. Ann N Y Acad Sci 1052: 233-242, 2005

2. Buga AM, Bălşeanu A, Popa-Wagner A and Mogoantă L: Strategies to improve post-stroke behavioral recovery in aged subjects. Rom J Morphol Embryol 50: 559-582, 2009.

3. Fisher M, Feuerstein G, Howells DW, et al; STAIR Group: Update of the stroke therapy academic industry roundtable preclinical recommendations. Stroke 40: 2244-2250, 2009.

4. Matsui T, Arai H, Yuzuriha T, et al: Elevated plasma homocysteine levels and risk of silent brain infarction in elderly people. Stroke 32: 1116-1119, 2001.

5. Lloyd-Jones D, Adams R, Carnethon M, et al; American Heart Association Statistics Committee and Stroke Statistics Subcommittee: Heart disease and stroke statistics - 2009 update: a report from the American Heart Association Statistics Committee and Stroke Statistics Subcommittee. Circulation 119: 480-486, 2009.

6. Dittmar M, Spruss T, Schuierer G and Horn M: External carotid artery territory ischemia impairs outcome in the endovascular filament model of middle cerebral artery occlusion in rats. Stroke 34: 2252-2257, 2003.

7. Longa EZ, Weinstein PR, Carlson S and Cummins R: Reversible middle cerebral artery occlusion without craniectomy in rats. Stroke 20: 84-91, 1989.

8. Boyko M, Zlotnik A, Gruenbaum BF, et al: An experimental model of focal ischemia using an internal carotid artery approach. J Neurosci Methods 193: 246-253, 2010. 
9. Durukan A and Tatlisumak T: Acute ischemic stroke: overview of major experimental rodent models, pathophysiology, and therapy of focal cerebral ischemia. Pharmacol Biochem Behav 87: 179-197, 2007.

10. Koizumi J, Yoshida Y, Nakazawa T and Ooneda G: Experimental studies of ischemic brain edema. I: a new experimental model of cerebral embolism in rats in which recirculation can be introduced in the ischemic area. Jpn J Stroke 8: 1-8, 1986.

11. Ma J, Zhao L and Nowak TS Jr: Selective, reversible occlusion of the middle cerebral artery in rats by an intraluminal approach. Optimized filament design and methodology. J Neurosci Methods 156: 76-83, 2006.

12. Durukan A, Strbian D and Tatlisumak T: Rodent models of ischemic stroke: a useful tool for stroke drug development. Curr Pharm Des 14: 359-370, 2008.

13. Prieto R, Carceller F, Roda JM and Avendaño C: The intraluminal thread model revisited: rat stain differences in local cerebral blood flow. Neurol Res 27: 47-52, 2005.

14. Cheung JS, Wang E, Lo EH and Sun PZ: Stratification of heterogeneous diffusion MRI ischemic lesion with kurtosis imaging evaluation of mean diffusion and kurtosis MRI mismatch in an animal model of transient focal ischemia. Stroke 43: 2252-2254, 2012

15. Chauveau F, Cho TH, Riou A, et al: Does acute behavioral testing reflect successful ischemia in rats with transient middle cerebral artery occlusion? Int J Stroke 7: 465-472, 2012.

16. Bederson JB, Pitts LH, Tsuji M, Nishimura MC, Davis RL and Bartkowski H: Rat middle cerebral artery occlusion: evaluation of the model and development of a neurologic examination. Stroke 17: 472-476, 1986.
17. Nagaoka A, Suno M, Shibota M and Kakihana M: Effects of idebenone (CV-2619) on neurological deficits, local cerebral blood flow, and energy metabolism in rats with experimental cerebral isehemia. Nihon Yakurigaku Zasshi 84: 303-309, 1984 (In Japanese).

18. Tatlisumak T, Takano K, Carano RA, Miller LP, Foster AC and Fisher M: Delayed treatment with an adenosine kinase inhibitor, GP683, attenuates infarct size in rats with temporary middle cerebral artery occlusion. Stroke 29: 1952-1958, 1998.

19. Hattori K, Lee H, Hurn PD, Crain BJ, Traystman RJ and DeVries AC: Cognitive deficits after focal cerebral ischemia in mice. Stroke 31: 1939-1944, 2000.

20. Airavaara M, Shen H, Kuo CC, et al: Mesencephalic astrocyte-derived neurotrophic factor reduces ischemic brain injury and promotes behavioral recovery in rats. J Comp Neurol 515: 116-124, 2009.

21. Boyko M, Ohayon S, Goldsmith T, et al: Morphological and neuro-behavioral parallels in the rat model of stroke. Behav Brain Res 223: 17-23, 2011.

22. White BC, Sullivan JM, DeGracia DJ, et al: Brain ischemia and reperfusion: molecular mechanisms of neuronal injury. J Neurol Sci 179: 1-33, 2000.

23. Kawamura S, Li Y, Shirasawa M, Yasui N and Fukasawa $H$ : Reversible middle cerebral artery occlusion in rats using an intraluminal thread technique. Surg Neurol 41: 368-373, 1994. 